\title{
Erratum To: Accounting for Behavioral Responses during a Flu Epidemic Using Home Television Viewing
}

\author{
Michael Springborn ${ }^{1 *}$, Gerardo Chowell ${ }^{2,3,4}$, Matthew MacLachlan ${ }^{5}$ and Eli P. Fenichel ${ }^{6}$
}

\section{Erratum}

After the publication of this work [1], we became aware of errors in the reported results in Table 1. A corrected version of this table appears below. The main error involved reporting results for the low SEL class in the high SEL class row, and vice versa. Other small errors in reported $p$-values in the final column of the table have also been corrected.

The only implication of correcting these errors for the results discussed in the paper is to strengthen rejection of one of the null hypotheses tested. In the original text we observed that, "During the intervention period, on average the high SEL group shows a response that is over 50 \% greater than that of the low SEL group." Furthermore we remarked that, "This difference is significant at the $5 \%$ level." This difference is in fact significant at the $1 \%$ level. We regret the error.

\begin{abstract}
Author details
'Department of Environmental Science \& Policy, University of California, 2104 Wickson Hall, One Shields Ave., Davis, CA 95616, USA. ${ }^{2}$ School of Public Health, Georgia State University, P.O. Box 3965, Atlanta, GA 30302-3965, USA. ${ }^{3}$ Division of International Epidemiology and Population Studies, Fogarty International Center, National Institutes of Health, 31 Center Dr, MSC 2220, Bethesda 20892-2220, Maryland, USA. ${ }^{4}$ Mathematical, Computational \& Modeling Sciences Center, School of Human Evolution and Social Change, Arizona State University, 900 S. Cady Mall, Tempe, AZ 85287-2402, USA. ${ }^{5}$ Department of Agricultural \& Resource Economics, University of California, 2116 Social Sciences \& Humanities, One Shields Ave., Davis, CA 95616, USA ${ }^{6}$ Yale School of Forestry and Environmental Studies, 195 Prospect St., New Haven, CT 06511, USA
\end{abstract}

Received: 22 August 2016 Accepted: 22 August 2016

Published online: 05 September 2016

\section{References}

1. Springborn M, Chowell G, MacLachlan M, Fenichel EP. Accounting for behavioral responses during a flu epidemic using home television viewing. BMC Infect Dis. 2015;15(1):21.

\footnotetext{
* Correspondence: mspringborn@ucdavis.edu

'Department of Environmental Science \& Policy, University of California, 2104

Wickson Hall, One Shields Ave., Davis, CA 95616, USA
} 
Table 1 Summary statistics for daily percentage deviation from the long-run mean ATV $\left(\Delta_{t}\right)$ for various demographic groups

\begin{tabular}{|c|c|c|c|c|c|}
\hline \multirow{2}{*}{ group } & & \multicolumn{4}{|c|}{ statistics for $\Delta_{t}$ within the intervention period $(\tau)$} \\
\hline & & range & mean & mean $=0$ ( $p$-value) & equal means within class ( $p$ - value) \\
\hline aggregate & & {$[-1.4 \%, 22.6 \%]$} & $13.6 \%$ & $<0.001$ & \\
\hline \multirow[t]{2}{*}{ age class } & children & {$[-4.7 \%, 46.2 \%]$} & $23.7 \%$ & $<0.001$ & \multirow{2}{*}{$<0.001$} \\
\hline & adults & {$[-6.5 \%, 21.8 \%]$} & $8.9 \%$ & $<0.001$ & \\
\hline \multirow[t]{3}{*}{ SEL class } & low & {$[-3.5 \%, 21.0 \%]$} & $11.3 \%$ & $<0.001$ & \multirow{2}{*}{$\begin{array}{l}\text { low-med: } 0.06 \\
\text { med-high: } 0.40\end{array}$} \\
\hline & medium & {$[0.4 \%, 32.1 \%]$} & $15.3 \%$ & $<0.001$ & \\
\hline & high & {$[-0.4 \%, 31.7 \%]$} & $17.5 \%$ & $<0.001$ & low-high: <0.01 \\
\hline \multirow[t]{2}{*}{ time of day } & daytime & {$[-3.7 \%, 30.7 \%]$} & $18.4 \%$ & $<0.001$ & \multirow{2}{*}{$<0.001$} \\
\hline & nighttime & {$[-4.1 \%, 17.0 \%]$} & $9.6 \%$ & $<0.001$ & \\
\hline
\end{tabular}

